\title{
Molecular Weight Control of Curdlan ( $\beta$-1,3-Glucan Polysaccharide) Provides Unique Polynucleotide Binding Properties ${ }^{\dagger}$
}

\author{
Kazuya Koumoto, Hideki Kobayashi, ${ }^{*}$ Masami MizU, Taro Kimura,** Kazuo SAKURAI, \\ Toyoki KunitaKe, and Seiji SHINKAI ${ }^{\dagger \dagger, * * *}$
}

\author{
Department of Chemical Processes and Environments, Faculty of Environmental Engineering, The University of Kitakyushu, \\ 1-1 Hibikino, Wakamatsu-ku, Kitakyushu 808-0135, Japan \\ *Arakawa Chemical Industries, Ltd., Technopark Oho, 5 Okubo, Tsukuba 300-2611, Japan \\ **Fukuoka Industrial Technology Center, Biotechnology and Food Research Institute, 1465-5 Aikawa, Kurume 839-0861, Japan \\ ${ }^{* * *}$ Department of Chemistry and Biochemistry, Graduate School of Engineering, Kyushu University, Fukuoka 812-8581, Japan
}

(Received October 3, 2003; Accepted February 24, 2004; Published May 15, 2004)

\begin{abstract}
Recently, we found that schizophyllan belonging to a $\beta$-1,3-glucan polysaccharide family forms macromolecular complexes with certain single-stranded polynucleotides. The specific complex formation is originated from the $\beta$-1,3-glucan skeleton in the main chain. In this paper, we have reported that curdlan, which is a $\beta$-1,3-glucan polysaccharide without a side chain glucose unit, shows unique poly $(C)$ binding properties different from those of schizophyllan; in the experiments using curdlan samples with the controlled molecular weight, the complexation ability appeared only in a limited " $M_{\mathrm{w}}$ range window". Stoichiometric analysis revealed that the " $M_{\mathrm{w}}$ range window" appears as a result of the competition between curdlan-curdlan self-association and curdlan-polynucleotide complexation. Furthermore, when the experiments were extended to other polynucleotides such as poly (A), poly $(\mathrm{dA})$ and poly $(\mathrm{dT})$, the " $M_{\mathrm{w}}$ range window" also appeared at their characteristic molecular weights. We believe, therefore, that curdlan can be applicable, in spite of its cheapness, as a precious functional material to gene technology.

[DOI 10.1295/polymj.36.380]

KEY WORDS Polysaccharide-Polynucleotide Complex / $\beta$-1,3-Glucan / Polynucleotide Molecular Weight / Binding Window / Curdlan / Schizophyllan /
\end{abstract}

In nature, lots of biopolymers such as proteins, polynucleotides and polysaccharides play critical roles in various biological processes. In such processes, accurate polymer-polymer interactions systematically regulate the biological functions. Recently, we found that schizophyllan, which is a natural and neutral polysaccharide produced by fungus Schizophyllum commune, can specifically form macromolecular complexes with certain single-stranded polynucleotides. ${ }^{1-3}$ Stoichiometric analysis revealed that the complex consists of one polynucleotide chain and two polysaccharide chains. ${ }^{2}$ Interestingly, the complex is dissoci- ated with a cooperative manner upon heating as being similar to DNA duplexes. ${ }^{1}$ To the best of our knowledge, this is the first example that the specific polysaccharide-polynucleotide interaction is experimentally evidenced.

Schizophyllan consists of a $\beta$-1,3-glucan main chain structure and every third glucose unit has a $\beta$ 1,6-glucoside side chain as shown in Figure 1a. ${ }^{4,5}$ In nature, it adopts a triple-stranded helical conformation $(t$-SPG). Norisuye et al. reported that $t$-SPG is dissociated into a single chain of schizophyllan (s-SPG) when it is dissolved in dimethylsulfoxide (DMSO).

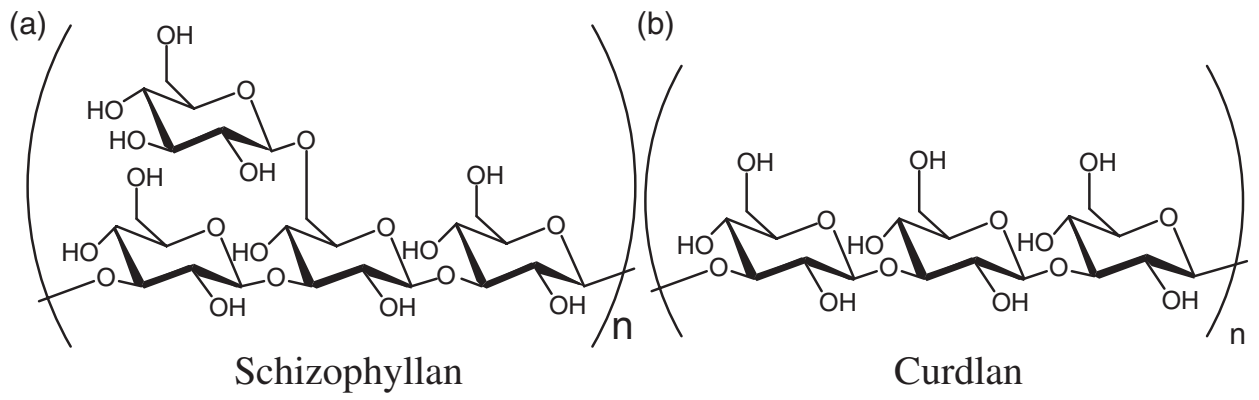

Figure 1. Repeating unit of polysaccharides: (a) schizophyllan and (b) curdlan.

\footnotetext{
${ }^{\dagger}$ This is the 16th paper in the series of "Polysaccharide-polynucleotide complexes".

${ }^{\dagger \dagger}$ To whom correspondence should be addressed (E-mail: seijitcm@mbox.nc.kyushu-u.ac.jp).
} 


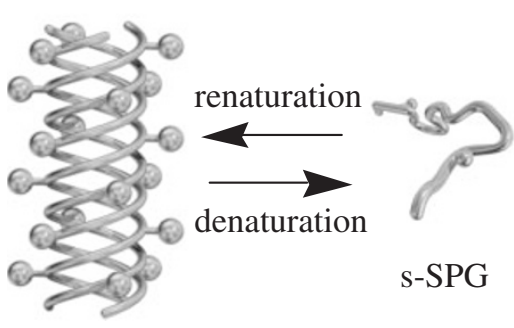

t-SPG

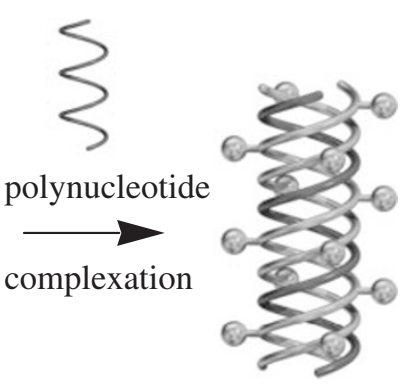

s-SPG/polynucleotide complex

Figure 2. Schematic illustration of the complex formation between polynucleotide and schizophyllan. In the illustration, light gray lines represent a schizophyllan chain and dark gray ones represent a polynucleotide chain.

The s-SPG chain can regain the triplex conformation by exchanging DMSO for water (renaturation). ${ }^{8,9}$ Our experiments have established that when the renaturation process is carried out in the presence of certain polynucleotides, the process creates a novel triple helix (the complex formation is schematically shown in Figure 2). In addition, it was also clarified that such complexation can occur commonly in water-soluble $\beta$-1,3-glucan polysaccharides such as lentinan and laminaran. ${ }^{10}$ Among the novel complexation properties, the following properties are considered to be particularly important in its practical applications; that is, (1) the thermal stability estimated from the dissociation temperature of the complexes is different in each polynucleotide, indicating that the complexation has a selectivity for each nucleobase $\mathrm{e}^{2,11}$ and (2) the complex formation between polynucleotides and polysaccharides can be easily controlled by changing the medium $\mathrm{pH}^{12}$ and adding the certain salt. ${ }^{13}$ Taking these properties into consideration, one can seriously consider an idea to apply the polysaccharide to the gene separation experiments. In fact, our preliminary result demonstrated that schizophyllan-appended polymer resin can act as a stationary phase for the polynucleotide separation. ${ }^{14}$ In order to separate nucleotides, very expensive and bio-degradable nucleotide derivatives have been used so far. In contrast, the polysaccharide-appended polymer resin can be used repeatedly without significant degradation of the appended-saccharide chains.

Among $\beta$-1,3-glucan polysaccharides, curdlan without a side chain glucose unit (see Figure 1b) is the most inexpensive and, therefore, has widely been used for various applications such as additives for food, paper and concrete, feeds for domestic animals and fishes, anti-HIV agents, immunoactivators, drug delivery vehicles, etc. ${ }^{15}$ Although curdlan is much less expensive than schizophyllan, its poor water-solubility has been an obstacle to apply it to biological purposes. Previously, we accidentally found that the water-solubility is improved with the decrease in the molecular weight and curdlan becomes possible to form complexes with polynucleotides as being similar to schizophyllan. ${ }^{16}$ In this full paper, we report our new findings, that is, unique polynucleotide binding properties of low-molecular-weight curdlan homologues obtained by fractionation of the molecular weight.

\section{EXPERIMENTAL}

\section{Materials}

Schizophyllan samples were kindly supplied by Taito Co., Ltd. (Kobe, Japan). Laminari-oligosaccharide mixture (degree of polymerization (DP): 8-15) and laminarihexaose were purchased from Seikagaku Corporation (Tokyo, Japan). Poly(C) (DP: 320), poly(A) (DP: 570), poly(dA) (DP: 300) and poly(dT) (DP: 260) were purchased from Amersham (NJ, USA). DMSO (spectroscopic grade) was purchased from Kishida Chemical Co., Ltd. (Osaka, Japan). All reagents were used without further purification. Low molecular-weight curdlan samples were synthesized according to the reported method. ${ }^{16}$

\section{GPC Analysis}

The molecular weights of the curdlan and schizophyllan samples were evaluated by gel permeation chromatography (GPC) using a TOSOH HLC-8020; apparatus two $\alpha-4000$ columns are connected in series, $\mathrm{LiBr}\left(0.02 \mathrm{~mol} \mathrm{dm}^{-3}\right) / \mathrm{DMF}$ was used as elution solvent and the instrument was calibrated by a polyethyleneoxide standard (TOSOH Co., Japan).

\section{Measurements and Instruments}

All samples were prepared by mixing a polynucleotide-containing water solution with a schizophyllancontaining DMSO solution, according to the reported method. ${ }^{2,11}$ This mixture was left at $4{ }^{\circ} \mathrm{C}$ for $3-10 \mathrm{~d}$ to allow the complexation to complete. Unless otherwise mentioned, the final concentrations were controlled as follows: 
$[\operatorname{poly}(\mathrm{C})]=2.5 \times 10^{-4} \mathrm{~mol} \mathrm{dm}^{-3} /$ monomer unit, [poly(A) $]=2.4 \times 10^{-4} \mathrm{~mol} \mathrm{dm}^{-3} /$ monomer unit, [poly $(\mathrm{dA})]=0.7 \times 10^{-4} \mathrm{~mol} \mathrm{dm}^{-3} /$ monomer unit, $[$ poly $(\mathrm{dT})]=0.7 \times 10^{-4} \mathrm{~mol} \mathrm{dm}^{-3} /$ monomer unit.

The concentrations of schizophyllan and curdlan were controlled to be a stoichiometric number to prevent the precipitation of excess high molecular-weight curdlan. The CD spectra were measured on a Jasco J$820 \mathrm{KS}$ spectropolarimeter in the $240-340 \mathrm{~nm}$ region using a $1 \mathrm{~cm}$ cell equipped with a water-jacket to control the cell temperature.

\section{RESULTS AND DISCUSSION}

Preparation of Low-molecular-weight $\beta$-1,3-Glucan Samples

Low-molecular-weight curdlan samples were prepared by acid hydrolysis according to the reported method $^{16}$ and those of schizophyllan was kindly supplied by Taito Co., Ltd. Their molecular weights $\left(M_{\mathrm{w}}\right)$ were evaluated by gel permeation chromatography (GPC) using polyethyleneoxide as a standard. The sample codes and the molecular weight data of schizophyllan and curdlan are summarized in Tables I and II, respectively.

\section{Molecular Weight Effect on the Complexation with $\operatorname{Poly}(C)$}

It is already known that the complex formation between $\beta$-1,3-glucan polysaccharides and poly $(\mathrm{C})$ indu-

Table I. The molecular weight data of schizophyllan

\begin{tabular}{crrc}
\hline Sample codes & \multicolumn{1}{c}{$M_{\mathrm{n}}$} & \multicolumn{1}{c}{$M_{\mathrm{w}}$} & $M_{\mathrm{w}} / M_{\mathrm{n}}$ \\
\hline SPG1 & 201,000 & 446,000 & 2.22 \\
SPG2 & 78,700 & 131,000 & 1.67 \\
SPG3 & 18,400 & 25,400 & 1.38 \\
SPG4 & 10,600 & 16,700 & 1.58 \\
SPG5 & 3,920 & 6,890 & 1.76 \\
SPG6 & 1,810 & 2,240 & 1.24 \\
SPG7 & 941 & 1,740 & 1.85 \\
SPG8 & 415 & 1,090 & 2.62 \\
\hline
\end{tabular}

Table II. The molecular weight data of curdlan

\begin{tabular}{crrc}
\hline Sample codes & \multicolumn{1}{c}{$M_{\mathrm{n}}$} & \multicolumn{1}{c}{$M_{\mathrm{w}}$} & $M_{\mathrm{w}} / M_{\mathrm{n}}$ \\
\hline CUR1 & $1,730,000$ & $2,320,000$ & 1.34 \\
CUR2 & 67,000 & 87,000 & 1.37 \\
CUR3 & 14,400 & 22,600 & 1.58 \\
CUR4 & 7,600 & 12,100 & 1.59 \\
CUR5 & 5,190 & 8,640 & 1.67 \\
CUR6 & 3,590 & 5,680 & 1.58 \\
CUR7 & 2,230 & 2,580 & 1.16 \\
LAM $_{\text {mix }}{ }^{\mathrm{a}}$ & 1,540 & 1,870 & 1.21 \\
LAM $(6)^{\mathrm{b}}$ & 914 & 982 & 1.07 \\
\hline
\end{tabular}

${ }^{\mathrm{a}} \mathrm{LAM}_{\text {mix }}$ : laminarioligosaccharide mixture $(\mathrm{DP}=8$ 15), ${ }^{b} \operatorname{LAM}(6)$ : laminarihexaose
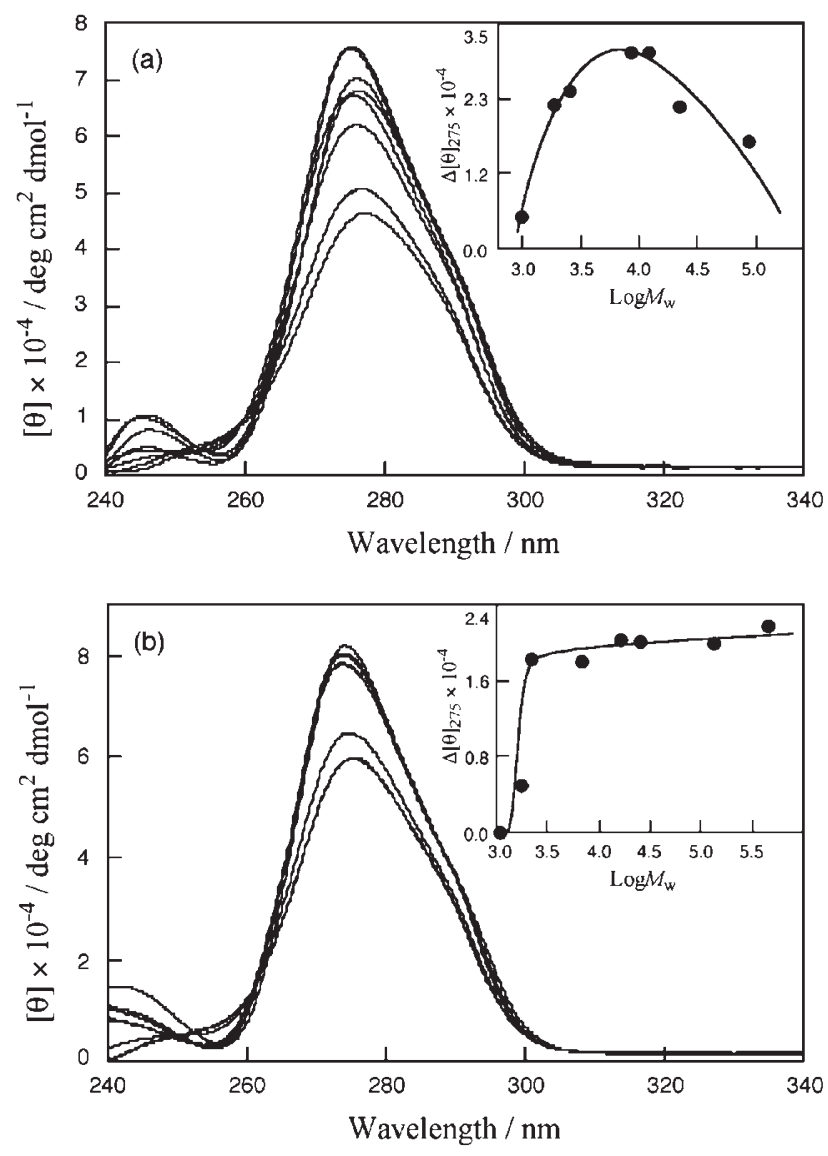

Figure 3. CD spectra for the mixtures of poly(C) and curdlan samples (a) and poly(C) and schizophyllan samples (b). Inset in the figure shows the relation between $\Delta[\theta]_{275}$ and the molecular weight of the polysaccharide.

ces characteristic changes in the circular dichroism (CD) spectrum; the $[\theta]_{275}$ value is enhanced by about $50 \%$ and a new band appears at around $245 \mathrm{~nm}$, where $[\theta]_{275}$ is the molecular ellipticity at $275 \mathrm{~nm}$. $^{1,10,16}$ Figure 3a compares the $\mathrm{CD}$ spectra for the mixtures of poly $(\mathrm{C})$ and curdlan samples at $5{ }^{\circ} \mathrm{C}$ and $V_{\mathrm{w}}=$ 0.75 , where $V_{\mathrm{w}}$ is the water volume fraction in the water-DMSO mixture. At $V_{\mathrm{w}}=0.75$, all curdlan samples are dissolved in the solution whenever the complex is formed with poly $(C)$. The clear enhancement of the $[\theta]_{275}$ value is observed for the mixtures of $\mathrm{LAM}_{\text {mix }} / \operatorname{poly}(\mathrm{C}), \quad \mathrm{CUR} 7 / \operatorname{poly}(\mathrm{C}), \quad \mathrm{CUR} 5 / \operatorname{poly}(\mathrm{C})$, CUR4/poly(C), CUR3/poly(C) and CUR2/poly(C), whereas the CD spectra for the mixtures of CUR1/ poly $(\mathrm{C})$ and $\operatorname{LAM}(6) / \operatorname{poly}(\mathrm{C})$ are identical with that of poly $(\mathrm{C})$ itself, indicating that CUR1 and $\operatorname{LAM}(6)$ cannot form the complex with poly $(\mathrm{C})$. We plotted the $\Delta[\theta]_{275}$ value corresponding to the increment of the cytosine stacking in poly $(\mathrm{C})$ against their molecular weight as shown in the inset in the figures. The enhancement of the $[\theta]_{275}$ value can be observed for the $\log M_{\mathrm{w}}$ range from 3 to 5 . In addition, the $[\theta]_{275}$ values result in a maximum at around 4 and become small in the lower and higher molecular weight range. 
On the other hand, Figure $3 b$ shows the $\mathrm{CD}$ spectra for the SPG/poly $(\mathrm{C})$ complexes. The enhancement of the $[\theta]_{275}$ value for the SPG/poly(C) complexes occur in the $\log M_{\mathrm{w}}$ above 3 and the value reaches a plateau in the $\log M_{\mathrm{w}}$ above 3.3. In contrast to the curdlan system, the decrease in the $[\theta]_{275}$ value in the higher molecular weight region is not observed for the SPG system.

To obtain a better insight into the molecular weight effect, we plotted the melting temperature $\left(T_{\mathrm{m}}\right)$ against their molecular weight. The $T_{\mathrm{m}}$ values were estimated from their $[\theta] v s$. $T$ melting curves according to a conventional method. ${ }^{17,18}$ As shown in Figure 4, a plot of $T_{\mathrm{m}}$ against their $\log M_{\mathrm{w}}$ shows an upward convex; the CUR4/poly(C) complex has the highest $T_{\mathrm{m}}$ value and the values become smaller as the $M_{\mathrm{w}}$ becomes higher or lower than that of CUR4. This feature is consistent with the relation observed for the $\Delta[\theta]_{275} \log M_{\mathrm{w}}$ plot (inset in Figure $3 a)$. It is undoubted, therefore, that curdlan possesses a unique poly $(\mathrm{C})$ binding property with a limited " $M_{\mathrm{w}}$ range window".

\section{Appearance of a Limited " $M_{w}$ Range Window"}

It is known that the thermal stability of the DNA duplex increases as the polymer chain becomes long. ${ }^{17}$ Therefore, one can easily understand the $M_{\mathrm{w}}$ effect observed for schizophyllan. However, the behavior observed for curdlan is different from that of DNA or schizophyllan. The unusual $T_{\mathrm{m}} v s . \log M_{\mathrm{w}}$ plot suggests, therefore, that some unexpected interaction inherent to the curdlan skeleton is operative in the higher $M_{\mathrm{w}}$ range. To clarify the reason why the curdlan/polynucleotide complexes become thermally unstable in the higher $M_{\mathrm{w}}$ range, we measured the Job plots for the curdlan/poly $(\mathrm{C})$ complexes as shown in Figure 5 , where $\Delta \mathrm{CD}_{275}$ is defined by Eq (1),

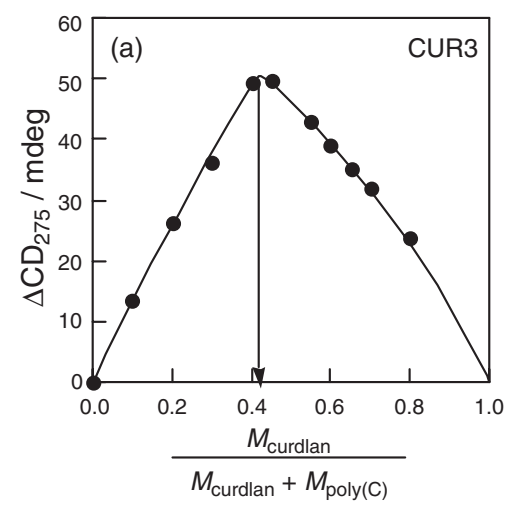

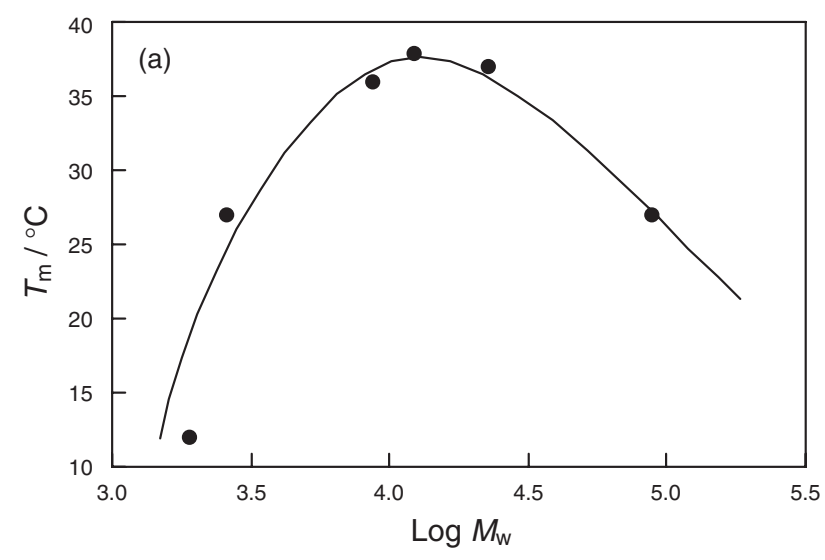

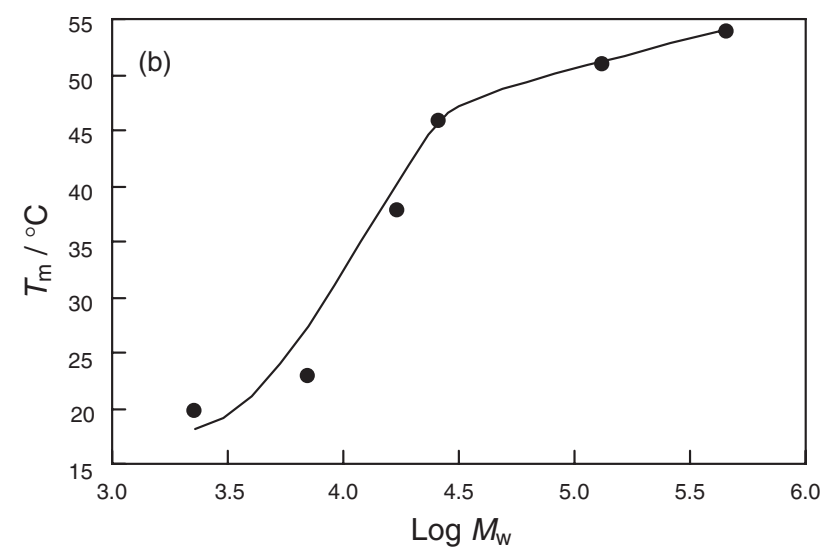

Figure 4. Plots of $T_{\mathrm{m}}$ of the poly(C) complexes as a function of the molecular weight of the saccharide chain: (a) curdlan and (b) SPG.

$$
\begin{aligned}
\Delta \mathrm{CD}_{275}= & \mathrm{CD}_{275}-\frac{M_{\text {curdlan }}}{M_{\text {poly }(\mathrm{C})}+M_{\text {curdlan }}} \\
& \times \mathrm{CD}_{275, \text { poly }(\mathrm{C})}
\end{aligned}
$$

where $\mathrm{CD}_{275}$ and $\mathrm{CD}_{275 \text {,poly(C) }}$ are the observed $\mathrm{CD}$ intensities at $275 \mathrm{~nm}$ for a mixture of poly(C) and curdlan and poly(C) itself, respectively, and $M_{\text {poly(C) }}$ and $M_{\text {curdlan }}$ are the molar concentrations of cytidine and the repeating unit of curdlan (to compare with schizophyllan, the repeating unit here denotes the

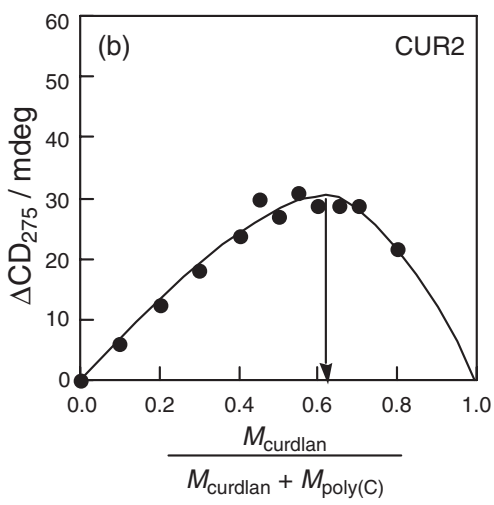

Figure 5. Job plots between curdlan (CUR2 (a) and CUR3 (b)) and poly(C): the sum of $[p o l y(C)]+[C U R 3$ or CUR2] was kept constant $\left(2.7 \times 10^{-4} \mathrm{~mol} \mathrm{dm}^{-3}\right)$. 

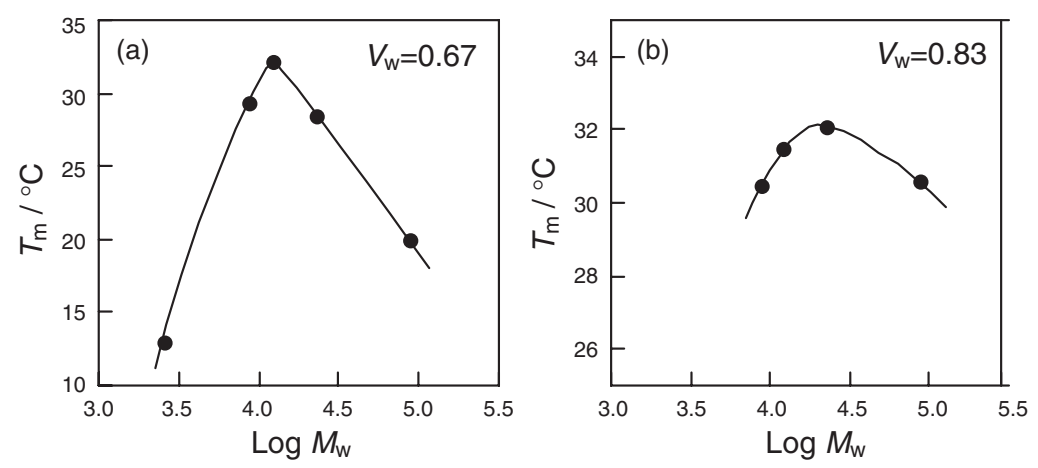

Figure 6. Plots of $T_{\mathrm{m}}$ of the curdlan/poly(C) complexes as a function of the molecular weight of curdlan: (a) $V_{\mathrm{w}}=0.67$ and (b) $V_{\mathrm{w}}=0.83$.

three glucose units in curdlan), respectively.

According to our previous studies, ${ }^{2,13}$ the polysaccharide-polynucleotide complexes have a specific stoichiometric number, 0.4. Figure 5a shows a plot of the $\triangle \mathrm{CD}_{275}$ against the molar ratio of curdlan for CUR3 as a typical example. We have found that the maximum for CUR3, CUR4, CUR5 and CUR7 all appears at 0.4 , which supports the view that, as in the SPG/ poly $(\mathrm{C})$ complexes, ${ }^{2}$ the curdlan complexes also consist of two curdlan chains and one poly $(\mathrm{C})$ chain. In contrast, the maximum for the CUR2/poly(C) complex appears at 0.6 (Figure 5b), which means that more glucose units of curdlan are used to bind to poly(C). CUR1, whose molecular weight is higher than CUR2, does not form the complexes but just precipitates after the self-assembling renaturation process. It is known that the high molecular-weight curdlan cannot dissolve in water to form a stable triplex. We consider, therefore, that the higher molecular-weight curdlan such as CUR1 is more hydrophobic and the curdlan-curdlan interaction overcomes the curdlan-poly $(\mathrm{C})$ interaction. CUR2 is a curdlan having a critical molecular weight, shifting from the curdlan self-association mode to the $\operatorname{poly}(\mathrm{C})$ complexation mode.

Then, we confirmed whether the " $M_{\mathrm{w}}$ range window" also appears at the different $V_{\mathrm{w}}$. According to our previous studies ${ }^{2}$ schizophyllan can form complexes with polynucleotides in the $V_{\mathrm{w}}$ range above 0.50 . Figure 6 shows the plots of $T_{\mathrm{m}}$ against their molecular weights at $V_{\mathrm{w}}=0.67$ and 0.83 . As shown in the figure, curdlan has a " $M_{\mathrm{w}}$ range window" in both $V_{\mathrm{w}}$ systems. Namely, the binding generally appears in the complexation behavior of curdlan and can be regarded to be a characteristic feature of curdlan.

\section{" $M_{w}$ Range Window" for Various Polynucleotides}

To investigate whether the " $M_{\mathrm{w}}$ range window" is also observed for other polynucleotides, we extended the measurements to poly(A), poly(dT) and poly$(\mathrm{dA}),{ }^{19}$ which are known to form complexes with
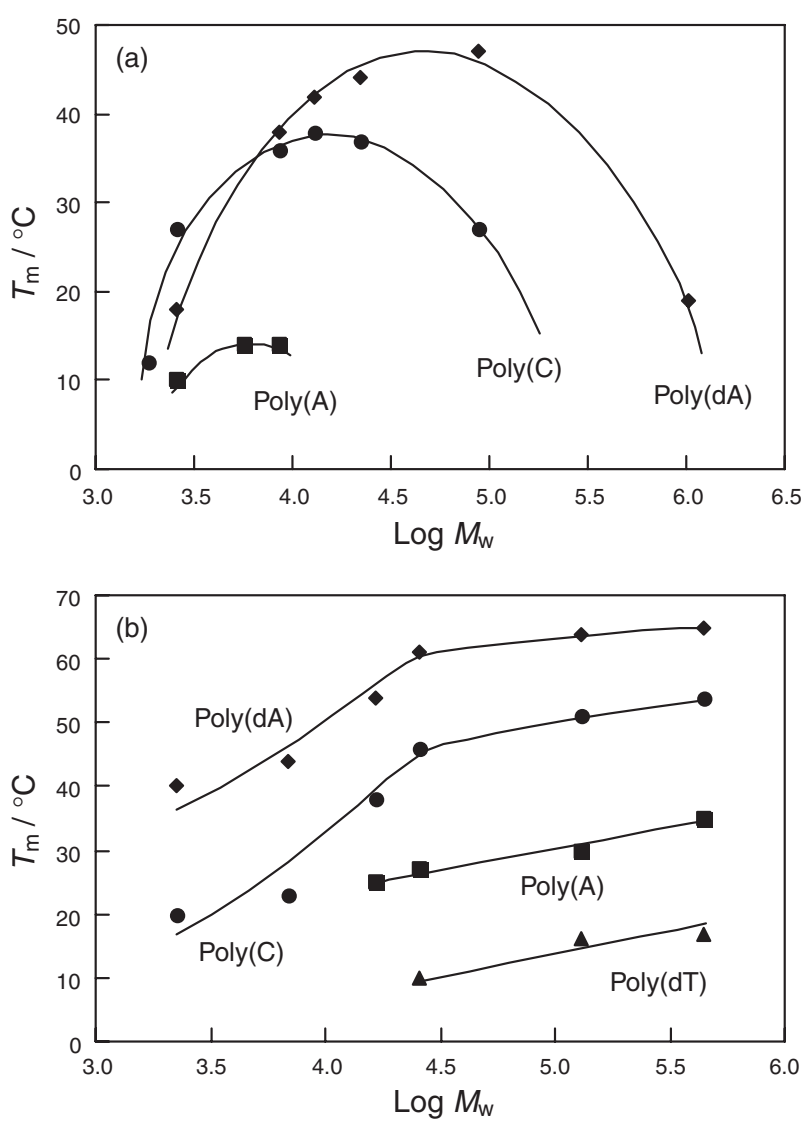

Figure 7. Plots of $T_{\mathrm{m}}$ of the polysaccharide/polynucleotide complexes as a function of the molecular weight of the saccharide chain: (a) curdlan and (b) schizophyllan.

schizophyllan. ${ }^{1,2}$ As a result, curdlan can also form complexes with poly(A) and poly(dA), whereas poly(dT) cannot. Figure 7 plots the $T_{\mathrm{m}}$ values of the complexes as a function of their molecular weight and compares them between curdlan and SPG. As can be seen, curdlan shows a " $M_{\mathrm{w}}$ range window" not only for $\operatorname{poly}(\mathrm{C})$ but also for both poly(A) and poly(dA), although the windows are somewhat narrower. On the other hand, the " $M_{\mathrm{w}}$ range window" has never been observed for SPG even though the $M_{\mathrm{w}}$ is increased up to 446,000 . The position of the 
" $M_{\mathrm{w}}$ range window" is characteristic for each polynucleotide; poly(A) can form complexes with curdlan in the $\log M_{\mathrm{w}}$ range from 3.4 to 4.0 and poly $(\mathrm{dA})$ from 3.4 to 6.0 .

\section{CONCLUSIONS}

We found, for the first time, that curdlan and schizophyllan show a different complexation ability for polynucleotides and only curdlan has a unique " $M_{\mathrm{w}}$ range window" specific to each polynucleotide. The stoichiometric number obtained from Job plots indicates that this window appears as a result of the competition between curdlan-curdlan self-association and curdlan-polynucleotide complex formation. We believe that these findings are useful to apply curdlan as a new functional material to gene technology: that is, commercially-available curdlan is really inexpensive but the appropriate molecular-weight control enables us to utilize it as a novel and precious DNA or RNA binder.

Acknowledgment. We thank Taito Co., Ltd. for providing the schizophyllan samples. K.K. is indebted to the Japan Society for the Promotion of Science (JSPS) for the financial support. This research has been conducted under the financial support from the JST SORST Program.

\section{REFERENCES}

1. K. Sakurai and S. Shinkai, J. Am. Chem. Soc., 122, 4520 (2000).

2. K. Sakurai, M. Mizu, and S. Shinkai, Biomacromolecules, 2 ,
641 (2001).

3. M. Mizu, T. Kimura, K. Koumoto, K. Sakurai, and S. Shinkai, Chem. Commun., 2001, 429.

4. S. Kikumoto, T. Miyajima, S. Yoshizumi, S. Fujimoto, and K. Kimura, J. Agric. Chem. Soc. Jpn., 44, 337 (1970).

5. S. Kikumoto, T. Miyajima, K. Kimura, S. Okubo, and N. Komatsu, J. Agric. Chem. Soc. Jpn., 45, 162 (1970).

6. T. Yanaki, T. Norisuye, and H. Fujita, Macromolecules, 13, 1462 (1980).

7. T. Norisuye, T. Yanaki, and H. Fujita, J. Polym. Sci., Polym. Phys. Ed., 18, 547 (1980).

8. B. T. Stokke, A. Elgsaeter, D. A. Brant, and S. Kitamura, Macromolecules, 24, 6349 (1991).

9. B. T. Stokke, A. Elgsaeter, D. A. Brant, T. Kuge, and S. Kitamura, Biopolymers, 33, 193 (1993).

10. T. Kimura, K. Koumoto, K. Sakurai, and S. Shinkai, Chem. Lett., 2000, 1243.

11. K. Koumoto, T. Kimura, M. Mizu, T. Kunitake, K. Sakurai, and S. Shinkai, J. Chem. Soc., Perkin Trans. 1, 2002, 2477.

12. K. Sakurai, R. Iguchi, M. Mizu, K. Koumoto, and S. Shinkai, Bioorg. Chem., 31, 216 (2003).

13. K. Sakurai, R. Iguchi, K. Koumoto, T. Kimura, M. Mizu, Y. Hisaeda, and S. Shinkai, Biopolymers, 65, 1 (2003).

14. T. Kimura, K. Koumoto, M. Mizu, K. Sakurai, and S. Shinkai, Chem. Lett., 2002, 1240.

15. I. Lee, "Biopolymers 5 -polysaccharides I-," ed. E. J. Vandamme, S. De Baets and A. Steinbüchel, Wiley-VCH, Weinheim, 2002, p 135.

16. K. Koumoto, T. Kimura, H. Kobayashi, K. Sakurai, and S. Shinkai, Chem. Lett., 2001, 908.

17. D. Pörschke, Biopolymers, 10, 1989 (1971).

18. V. V. Filimonov and P. L. Privalov, J. Mol. Biol., 122, 465 (1978).

19. Although we also mixed poly $(\mathrm{G})$ with curdlan samples, no complexation could be observed. 